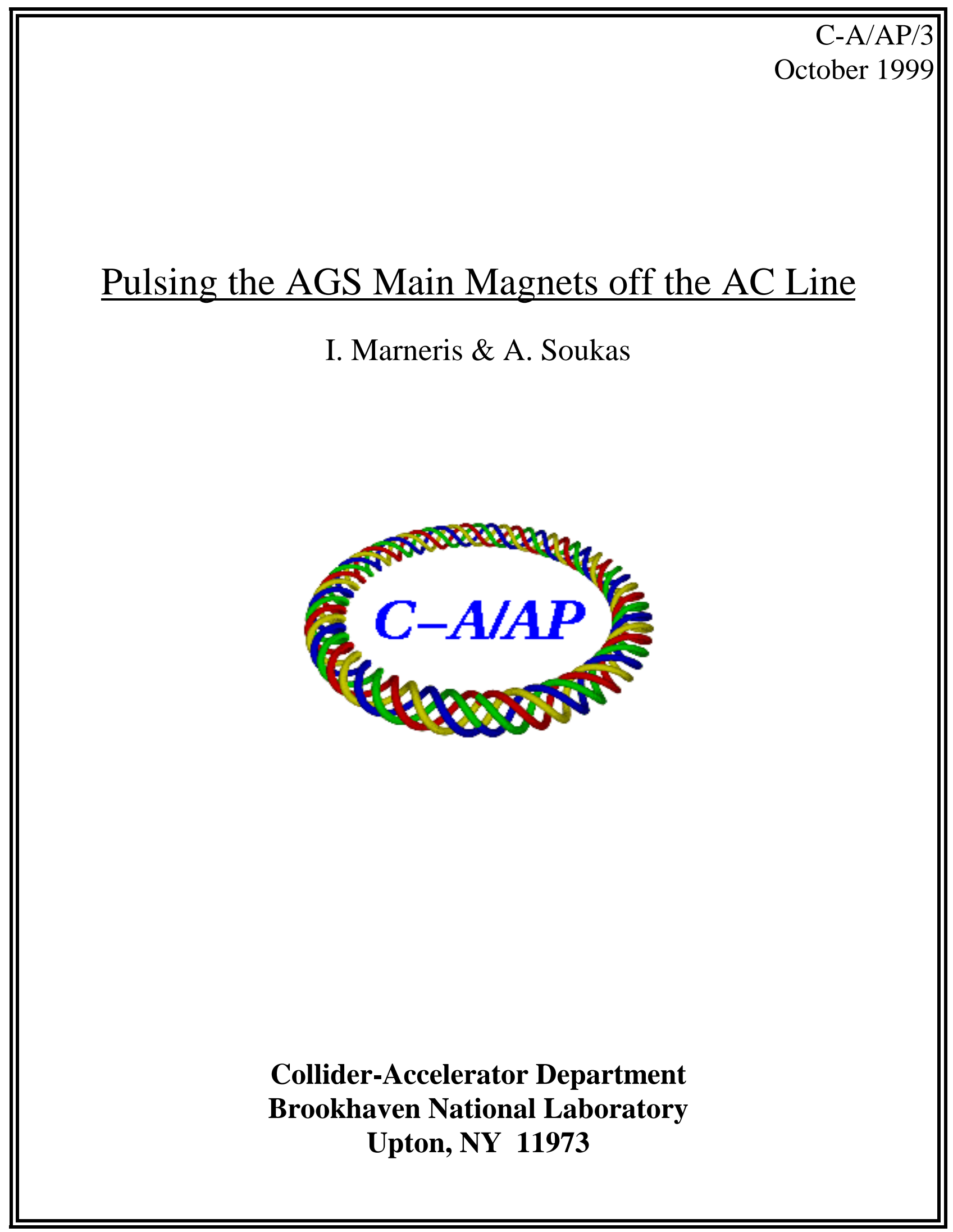




\title{
PULSING THE AGS MAIN MAGNETS OFF THE AC LINE
}

\author{
I. MARNERIS \& A. SOUKAS
}

October 6,1999

\section{I- INTRODUCTION}

For certain applications it is desirable to be able to run the AGS without using either of the two available motor-generator sets, i.e. the Siemens or the Westinghouse. Besides the obvious economic benefits of lower operating power losses and operation without personnel or specialists on shift, this mode reduces the wear and tear on the mechanical rotating equipment and enables more time for short-term or routine maintenance to be conducted on the rotating equipment.

Thus, we have implemented a scheme in which we can run the AGS Main Magnets (MM) directly off the incoming utility power line. This scheme is by necessity limited in power and hence only low energy operation of the AGS is possible. The limitations are set by cost and power line disturbances.

\section{II- IMPLEMENTATION}

The powering scheme utilizes a transformer from an old decommissioned AGS substation $(7 \mathrm{~N})$ as its basis. The transformer is a normal power distribution type with a $2.58 \mathrm{MVA}$ rating (65 degrees $\mathrm{C}$ rise) and a step-down voltage ratio of $13.8 \mathrm{kV} / 2.4 \mathrm{kV}$. The $2.4 \mathrm{kV}, 600 \mathrm{Amp}$ secondary output is connected to feed the usual AMMPS transformers and controlled rectifiers. Other than the by-passing of some low-level interlocks associated with either of the two MG sets, which are not required since the MG sets are not used, no other changes to the normal AMMPS equipment need to be made.

Physically, the ac powering scheme consists of a $13.8 \mathrm{kV}$ fused disconnect switch, the 2.5 MVA transformer (7N), and a 600 Ampere contactor that is recycled from the old AGS RF system. These are installed in the B928/929 substation yard. Cables (750 MCM) are run from the contactor output to the normal Siemens $52 \mathrm{G}$ circuit breaker located in the Siemens basement, where the generator bus feeds of both the Siemens or the Westinghouse are disconnected. The rest of the Siemens P-bank and F-bank transformers and silicon-controlled rectifiers remain intact. The PLC controls and operations computer interface remain intact. The contactor is manually controlled ON/OFF from the Siemens Control Room and is equipped with normal ac overload relay protection. 


\section{III- RATINGS, TESTING \& OPERATION}

The AGS, at times, due to its versatility and the successful operation of its Booster, is used to run experiments for Experimental Users at low energies. In particular, the acceleration of $\mathrm{Fe}+26$ heavy ions to $\sim 1 \mathrm{Gev/nucleon}$, is used by NASA Experimenters for physics and cell/biological effects studies.

The main magnetic field required in the AGS ring dipoles is approximately 1.5 KGauss which translates to a current of 660 Amperes dc from the MMPS, pulsed at a repetition rate of approximately 2.5 seconds. It has been desired for quite some time to provide low power cycles such as this from the ac line. However, costs were considered too high. Recently a power transformer with the approximate proper ratings had become available from a decommissioned AGS substation. For a minimum cost, consisting mostly of electrical labor, it was decided to set up this transformer in the scheme described earlier.

The desired cycle for the NASA Experiments, obtained from an archived file, is shown in Figure 1. The power was derived from the Siemens MG set using only the F Bank transformers and rectifiers.

A plan was devised to test the operation of the installed system into the AGS MM at the end of the present FY 99 program while the AGS ring was still locked up. The proper hookups were made and the unneeded MG set interlocks were by-passed. The testing began on Tuesday August 31 and concluded on Wednesday September 1, 1999.

The test cycle was constructed using the standard AGS MMPS computer application program after the proper adjustment of the program parameters. The AMMPS can employ its $\mathrm{P}$ bank (pulse) or its F bank (flattop) Siemens transformers to produce any given cycle. Since the P banks provide too much voltage and the application program does not have the flexibility to switch between banks effectively, it was decided to employ only the F banks to create a NASA type cycle. The final cycle is shown in Figure 2 and was saved as library file (B(t)/TRANS_FBANK_IM). The total cycle time is less than 2.4 seconds and further optimization can be performed. Figure 2 shows the current reference and current outputs, as well as the calculated voltage reference and output voltage of one of the F Banks. This voltage is at the rectifier unfiltered output. The actual magnet voltage is attenuated by about a factor of 10 by the passive filter and 24-pulse output.

Using the P Banks under large phase-back conditions, generates a large amount of reactive power. Since this must be supplied by the ac line transformer we found that the line current was excessive and thus, USING THE P BANKS SHOULD BE AVOIDED.

The following lists the maximum values of the ac line scheme:

$$
\begin{aligned}
& \text { Maximum Power------------------2.5 MVA } \\
& \text { Maximum Line Current------------600AMP } \\
& \text { Maximum F Bank dc Voltage------700VOLT }
\end{aligned}
$$




\section{Maximum P Bank dc Voltage------3500VOLT \\ For dc Operation, F Bank----------3000AMP Maximum dc Current \\ For Pulsed Operation, P Bank--- 100MSEC of Accel up to 650AMP}

\section{IV- OTHER USES}

The ac source described above also can be used during the beginning of AGS running periods or startups, where injection/capture/acceleration tuneups and optimizationa can be conducted. These periods usually can last typically up to 1-2 weeks. Also, Booster extraction studies, AGS injection studies, or Instrumentation studies and development, can be accomplished at a minimum cost.

At power levels up to the 2.5 MVA, voltage flicker of the ac line should not be a problem. Calculations show that even higher power levels $(2 \mathrm{X})$ can be absorbed by the L.I. power distribution without deleterious effects.

\section{V- ACKNOWLEDGEMENT}

We want to express our appreciation to the following AGS individuals who contributed greatly to this effort: M. Bannon, R. Bonati, D. Bastedo, J. Spinner, and M. Clancy. 